\title{
Nonintubated anatomical segmentectomy: does the benefit overcome the risks? - a narrative review
}

\author{
Carlos Galvez $^{1}$, Sergio Bolufer ${ }^{1}$, Juan Manuel Córcoles $^{2}$, Francisco Lirio ${ }^{1}$, Julio Sesma ${ }^{1}$, Andres Obeso ${ }^{3}$ \\ ${ }^{1}$ Thoracic Surgery Department, Hospital General Universitario Alicante, Alicante, Spain; ${ }^{2}$ Thoracic Surgery Department, Hospital Del Vinalopo, \\ Alicante, Spain; ${ }^{3}$ Thoracic Surgery Department, Hospital Clinico Universitario de Santiago, Santiago de Compostela, Spain \\ Contributions: (I) Conception and design: C Galvez; (II) Administrative support: All authors; (III) Provision of study materials or patients: All authors; \\ (IV) Collection and assembly of data: All authors; (V) Data analysis and interpretation: C Galvez; (VI) Manuscript writing: All authors; (VII) Final \\ approval of manuscript: All authors.
}

Correspondence to: Carlos Galvez, MD, PhD. Thoracic Surgery Department, Hospital General Universitario Alicante; C/Pintor Baeza, 12, 03010, Alicante, Spain. Email: carlos.galvez.cto@gmail.com.

Objective: Search the evidence available regarding nonintubated anatomical segmentectomies and discuss about outcomes.

Background: Nonintubated thoracic surgery has evolved since initial "rescue procedures" for highrisk patients under awake anesthesia, until sedative nonintubated resections including anatomical segmentectomies. Nonetheless, very few experienced centers in Asia have collected most of this short caseload worldwide, thus reflecting the difficult advance in this very specific minimally-invasive procedure. In fact, the evidence about nonintubated segmentectomies suggests only little benefits in terms of postoperative recovery (chest tube duration, hospital stay).

Methods: We review comparative studies, randomized trials, systematic reviews and meta-analysis about nonintubated segmentectomies since its first report. We focused in some controversial aspects and which the conclusions should be.

Conclusions: Evidence regarding the potential benefit of nonintubated segmentectomies is really poor nowadays due to very few studies reported from just highly-experienced centers. The evidence points to a better postoperative recovery in terms of chest tube duration and hospital stay, but these advantages are limited by non-randomized design and methodological biases. Nonetheless the standard with general anesthesia and orotracheal intubation is highly safe in the low-risk cohort of patients strictly selected for nonintubated segmentectomies, so it seems that the expected advantages of this anesthetic method have not been reached or at least not been assessed, especially those regarding respiratory complications and stress biomarkers. Efforts should be made in order to clarify the concepts and the targeted population, homogenize the anesthetic methods, design randomized trials assessing the variables where the expected benefits can be measured, and state conclusion according to these outcomes.

Keywords: Sublobar resection; nonintubated surgery; awake thoracic surgery

Received: 02 June 2021; Accepted: 06 August 2021; Published: 20 October 2022.

doi: 10.21037/jovs-21-33

View this article at: https://dx.doi.org/10.21037/jovs-21-33

$\wedge$ ORCID: 0000-0002-1226-0841. 


\section{Introduction}

Anatomical segmentectomy has been considered for many years a rescue resection procedure only for high risk patients deemed inoperable for lobar resection due to decreased pulmonary function or comorbidity (1). However, as minimally invasive thoracic surgery developed, the idea of lung-sparing resection became more attractive for thoracic surgeons, especially due to advances in early diagnosis and lung cancer screening programs already available in some countries (2-4). Many studies show evidence that anatomical segmentectomies are oncologically correct for some subtypes of early stage non-small cell lung cancer (NSCLC) (5-8), but their indications are also extended to benign or metastasic central lesions, or even multifocal or recurrent primary cancers (9).

Nonintubated anatomical resections under spontaneous breathing in low-risk patients have been performed but are limited to a few highly experienced centers mainly in Asian countries (10-12). There are still many concerns about indications, technical details, and potential advantages and risks that should be first addressed (13).

Combination of minimally invasive surgical techniques (uniportal video-assisted thoracic surgery (VATS), subxiphoid VATS), lung-sparing resections and less aggressive anesthesia is promising, but assessing the short evidence available should make us think: Do the benefits overcome the potential risks while comparing to a highly-safe method as orotracheal intubation and mechanical ventilation?

In this narrative review, we will bring to discussion some key points. We present the following article in accordance with the Narrative Review reporting checklist (available at https://jovs.amegroups.com/article/view/10.21037/jovs$21-33 / \mathrm{rc})$.

\section{Methods}

On 2020, we published a review article about Nonintubated anatomical segmentectomies (14) collecting all available evidence about these resections under spontaneous breathing. We also detailed the indications, exclusion criteria, and technical aspects to deal with during nonintubated anatomical segmentectomies.

Searching PubMed and Cochrane Library since 2019 when we wrote the manuscript (14), shows that only 3 new results have been published regarding nonintubated anatomical segmentectomy: one meta-analysis (15), one comparative retrospective study (11), and one case series (16). This little evidence means that there have been very few advances on this combination of minimally invasive techniques. So, the questions are: is the interest in nonintubated anatomical resections decreasing? Are the results discouraging? Do the benefits overcome the risks compared to conventional intubation and mechanical ventilation?

In this review of PubMed and Cochrane Library (using the terms: "nonintubated+sublobar", "non-intubated+sublobar", "awake+sublobar", "nonintubated+segmentectomy", "nonintubated+segmentectomy", "awake+segmentectomy") we will make a comprehensive analysis of studies published or accepted ahead of print, in English, since first report in 2012 (17), and we will limit the review to comparative studies (both prospective and retrospective), randomized trials and systematic reviews/meta-analysis. We will try to answer specific controversial questions:

(I) Are nonintubated anatomical segmentectomies feasible?

(II) Are nonintubated anatomical segmentectomies safe?

(III) Are nonintubated anatomical segmentectomies oncologically safe?

(IV) Are the benefits what we are looking for?

\section{Are nonintubated anatomical segmentectomies feasible?}

The answer is YES. The first specific series of nonintubated anatomical segmentectomies was published in 2013 (12) but there are reports from the same authors since 2012 with a series including 16 cases (17). Since then, several more case series and case reports have been published (18-20).

There are no specific randomized trials comparing nonintubated versus intubated VATS segmentectomies over the last 9 years since the first specific series was published, and only few comparative studies. First was published in 2016 (21) reporting their 5-year experience of 140 segmentectomies by multiportal VATS, which included 48 segmentectomies performed under epidural anesthesia and laryngeal mask, sedation and vagal block.

The same surgical team also published in 2016 a propensity score matching analysis of early outcomes on VATS for NSCLC regarding the type of anesthesia (22). Three hundred sixty-three cases of lobectomy or anatomical segmentectomy within a 3-year period were included. They excluded 24 patients, and finally 151 patients were operated under nonintubated VATS, including 32 anatomical segmentectomies. They performed a propensity score matching, final analysis included 20 nonintubated and 20 
intubated anatomical segmentectomies.

In 2021 (16), probably the most experienced surgical team in nonintubated VATS, from Taiwan, has published a retrospective analysis of a 4-year period [2014-2018] comparing 32 nonintubated uniportal versus 62 nonintubated multiportal segmentectomies. The comparison focused on the surgical approach but not the anesthetic method. This same group from Taiwan, published the actual year (11), a retrospective analysis of 185 consecutive uniportal VATS anatomical segmentectomies in a 5-year period [2014-2019], 50 of them were nonintubated. They performed a propensity score matching of 43 cases in each arm.

Reader can easily identify that only two highlyexperienced centers from Taiwan and China have reported all the comparative studies and almost the totality of nonintubated segmentectomies within almost a 10 -year period, so the relevant question is: is it feasible out of highvolume centers in Asian?

It is well known that nonintubated anatomical resections are performed after careful selection criteria. In the most recent analysis from Liu and colleagues (16), we should highlight that between $67.7-90.6 \%$ of the patients were women, with body mass index (BMI) between 21.9 (uniportal) and 22.1 (multiportal), and mean preoperative FEV1 exceeded $100 \%$. The type of segmentectomy was predominantly from upper lobes or simple segments of lower lobes, with almost none complex segmentectomy from basilar segments. All this data means that nonintubated approach was strictly selected for a very specific subset of lung cancer patients, but does this subset of patients allow most Thoracic Surgery departments to perform this approach in a conventional manner? In 2017, Salati et al. published data from the European Society of Thoracic Surgery (ESTS) database, with 62,774 lung cancers, including 51,931 primary lung cancers. Only one third of patients were women, mean BMI was 25.8, and median ppoFEV1 is $73.3 \%$, which reflects a very different lung cancer patient's profile (23).

Some attempts from Europe have also been published $(18,20,24)$, but have not gained continuity and have not spread thus reflecting the complexity.

\section{Are nonintubated anatomical segmentectomies safe?}

If we look at data published from the propensity score matching of 43 nonintubated and 43 intubated segmentectomies by Liu et al. (11), there were no conversions to intubation or thoracotomy, and no significant differences in conversion to multiportal VATS due to extense adhesions ( $4.7 \%$ vs. $2.3 \%, \mathrm{P}=0.557)$. There were also no differences in lowest oxygen saturation measured by oximetry $(97.1 \pm 3.7$ vs. $97.2 \pm 3.7, \mathrm{P}=0.743)$, but what is most important no significant differences were noted in peak end tidal carbon dioxide during procedures $(42.9 \pm 7.5 \mathrm{vs}$. $43.6 \pm 4.8, \mathrm{P}=0.633)$. There were no significant differences in postoperative complications and parameters such as chest tube duration and length of stay were comparable.

In the comparison of 48 nonintubated segmentectomies with 92 intubated of the other highly experienced team (21), mean peak $\mathrm{EtCO}_{2}$ was clearly higher in the nonintubated group (44.81 vs. $33.15 \mathrm{mmHg}, \mathrm{P}<0.001$ ), but there was only one conversion to intubation $(2.1 \%)$. There were no significant differences in lowest oxygen saturation, blood loss or conversion to thoracotomy, and postoperative complications were comparable between both groups.

This is the only comparative evidence available, and if we just read, the results indicate that nonintubated segmentectomy is safe. But if we make an exhaustive analysis, we can point out some interesting data. The most important feature is the patient's profile in these series: very low BMI (21.59 in Guo's paper and 22.1 in Liu's paper), excellent preoperative pulmonary function measured by FEV1 (95.09\% of predicted in Guo's and 112.2\% of predicted in Liu's paper) and low proportion of chronic obstructive pulmonary disease (COPD) patients $(0 \%$ in Guo's paper and $2.3 \%$ in Liu's paper). So nonintubated segmentectomy in highly experienced centers proved to be safe for a very strictly selected cohort of patients without chronic obstructive pulmonary disease, with excellent pulmonary function tests and very low BMI. This is probably the most important limitation for spread of nonintubated segmentectomy within Thoracic Surgery centers.

But surgeons who have dealt with nonintubated anatomical resections we all know that when you try to perform this approach in patients not complying these features, peak end-tidal $\mathrm{CO}_{2}$ considerably raises and mediastinal excessive movement jeopardizes the successful performance and increases the risk of conversion to intubation and mechanical ventilation. In fact, Wang et al. from Taiwan (25), published their results in lung cancer patients with impaired pulmonary function defined as preoperative FEV1 less than 70\%, American Society of Anesthesiologists physical status score of 4 or less, but with $\mathrm{BMI}$ less than 30, without evidence of pleural adhesions and 
Table 1 Lymph node assessment during nonintubated surgery: comparative studies

\begin{tabular}{|c|c|c|c|c|c|}
\hline Author & Year & Variable & Nonintubated group (n, \%) & Intubated group (n, \%) & $P$ value \\
\hline \multirow[t]{2}{*}{ Liu et al. (22) } & 2016 & - Mean no. of lymph nodes & $7.8 \pm 5.4$ & $6.4 \pm 5.3$ & 0.412 \\
\hline & & - Mean no. of lymph node stations & $3.2 \pm 1.4$ & $2.7 \pm 1.5$ & 0.286 \\
\hline Liu et al. (11) & 2021 & - Mean no. of lymph nodes & $4.3 \pm 3.4$ & $5.7 \pm 4.1$ & 0.127 \\
\hline
\end{tabular}

without anticipated difficult airway. They operated on 28 patients, including 3 segmentectomies and 4 lobectomies. There was one case of conversion to tracheal intubation (4\%) due to persistent intraoperative wheezing and difficult breathing. During the postoperative, $18 \%$ presented air leak longer than 5 days, and $21 \%$ subcutaneous emphysema, with $7 \%$ of acute exacerbation of COPD. Postoperative chest tube duration and hospital stay were 3 and 6 days respectively. Four patients (14\%) required hospital readmission, and only $50 \%$ of patients were free from anesthetic or postoperative complications, thus reflecting very different outcomes when compared to strictly selected cases.

Conclusion should be that nonintubated segmentectomy is safe in a very specific subset of patients.

\section{Are nonintubated segmentectomies oncologically safe?}

When dealing with oncological surgery regarding lung cancer, we should not only assess safety of the procedure in terms of complications, but also analyze if the procedures can be performed accomplishing the standards recommended by the International Association for Study of Lung Cancer (IASLC) (26), and main guidelines (ESMO, NCCN) $(9,27)$.

There is no available data regarding overall and diseasefree survival in comparative studies, so conclusions can not be established. The reason for this is probably the limited evidence, with very few studies and cases, but also that the main purpose of surgeons dealing with nonintubated approach is still to demonstrate the feasibility and safety of nonintubated procedures more than its oncological impact.

Lymph node dissection could potentially be threatened by a mobile surgical field during nonintubated segmentectomies due to mediastinal movement or accidental cough. None of the comparative studies have found significant differences in terms of number of lymph nodes and stations during lymphadenectomy $(11,21,22)$ (Table 1). This reflects that if the patient and the case have been strictly selected, vagal blockade and regional anesthesia provides the surgeon a surgical field comparable to intubated patients, so lymph node dissection should not be compromised by the anesthetic method.

\section{Are the benefits what we are looking for?}

Nonintubated thoracic surgery, or as it was first attempted awake thoracic surgery, initially tried to give a surgical chance for inoperable or high-risk patients (28-30) in the field of lung-volume reduction surgery (LVRS), secondary spontaneous pneumothorax, resection of peripheral nodules $(31,32)$ and other procedures. The expected benefit was the successful surgical performance in high-risk patients, but there are no reports of nonintubated segmentectomies in these high-risk patients despite isolated cases (25). Table S1 summarizes main outcomes of awake thoracic surgery in high-risk patients.

But the focus quickly shifted when anatomical resections were attempted. Interest in Asian countries for nonintubated surgery was born in order to avoid orotracheal intubation in patients with smaller caliber tracheas where risk of tracheal injury was higher $(10,12,17)$, so these surgeons were not looking for operating inoperable or high-risk patients, but changing the standards of anesthesia to avoid tracheal injury. Thus, they evolved into less invasive surgical techniques (uniportal VATS) and preserving parenchyma resections (sublobar).

The question is: What is the risk of tracheal injury with double-lumen orotracheal tubes? A systematic review in 2020 (33) with 187 airway ruptures secondary to doublelumen tubes showed that $61 \%$ of the patients were women and the mean BMI was $22.8 \mathrm{Kg} / \mathrm{m}^{2}$, but it's really difficult to assess which proportion of double-lumen intubations 
Table 2 Respiratory complications in comparative studies

\begin{tabular}{|c|c|c|c|c|c|}
\hline Author & Year & Variable & Nonintubated group, n (\%) & Intubated group, n (\%) & $P$ value \\
\hline \multirow{4}{*}{ Guo et al. (21) } & & - Atelectasis & 0 & $2(2.2)$ & 0.546 \\
\hline & & - Sputum retention + bronchoscopy & 0 & $2(2.2)$ & 0.546 \\
\hline & & - Chylothorax & $1(2.1)$ & $1(1.1)$ & $>0.99$ \\
\hline & & - Subcutaneous emphysema & 0 & $1(1.1)$ & $>0.99$ \\
\hline Liu et al. (22) & 2016 & - Respiratory complications & 2 & 2 & 1.00 \\
\hline \multirow[t]{3}{*}{ Liu et al. (11) } & 2021 & - Prolonged air leak & $1(2.3)$ & $4(9.3)$ & 0.167 \\
\hline & & - Air embolism & 0 & 0 & \\
\hline & & - Chylothorax & 0 & 0 & \\
\hline
\end{tabular}

in the clinical practice complicates with tracheal injury. In conventional European or north American patients' double lumen orotracheal intubation tracheal injury is really uncommon in experienced hands, so the profit margin seems minimal. This advantage is of particular importance when we deal with this specific subset of small size trachea, more than a general benefit.

Tacconi and colleagues (34) hypothesized that thoracic surgery under spontaneous breathing might trigger lower levels of stress hormones and inflammation biomarkers, and that could be of main importance in terms of oncological disease. But most publications don't analyze these parameters, just report some outcomes regarding lower white cell blood count (21), but there are no analysis about blood cortisol levels, T-lymphocytes and natural killer cells (NK cells) populations count or inflammation markers such as cytokines. For this reason, no conclusions about its biological impact can be obtained.

There is no evidence that segmentectomies under spontaneous breathing are safer in terms of postoperative complications $(11,21)$, in fact the evidence suggesting this has been obtained from reports of lobectomies (15), probably because they are the most conventional anatomical resections.

And if we specifically look at respiratory complications, the evidence from comparative studies suggests that there are no significant differences (Table 2). If thoracic surgery under spontaneous breathing diminishes ventilator-induced damage such as barotrauma, volutrauma and atelectrauma, which is the reason for these comparable outcomes? Probably performing the procedures in strictly selected patients with excellent pulmonary function tests, makes finding this protection of lung damage almost a crusade.

Thus, which have been the observed benefits of nonintubated sublobar resections? If we look at the comparative studies, we can summarize that nonintubated patients had shorter time until resumption of oral intake, shorter anesthesia time and costs, shorter chest tube duration and postoperative hospital stay $(11,21,22)$ (Table 3).

Making a critical analysis as surgeons, we all know that these are parameters we can push forward if we are really enthusiastic on it, as it occurs with uniportal or subxiphoid VATS approach.

Which could be the explanation for improvement of chest tube duration or postoperative hospital stay if postoperative complications and pain do not significantly differ? All these advantages may fit into the concept of enhanced recovery after surgery (ERAS) programs more than real benefits of a different anesthetic performance.

\section{Discussion: key aspects for the future}

After this objective and critical analysis, it seems that nonintubated segmentectomies have not enough evidence after years since its first report, but conclusions can be interpreted in a different way. 
Table 3 Observed advantages in comparative studies

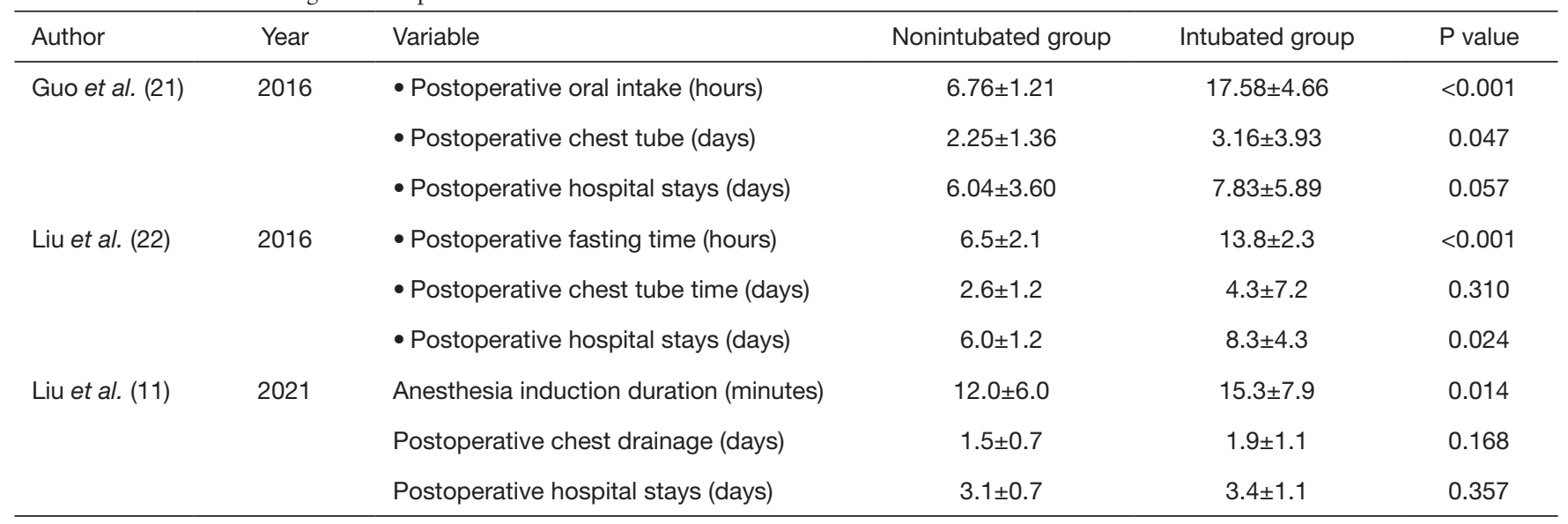

There are some key points that surgeons interested on nonintubated procedures should reconsider before starting, and experienced surgeons must focus on:

(I) Definition of two different scenarios. We must differentiate between operating highrisk or inoperable patients under spontaneous breathing to give them a surgical chance (usually awake procedures), from operating elective "ideal patients" for pulmonary anatomical resections, where we look for decreasing anesthetic aggression in order to accomplish a more "sustainable" surgery (usually non-awake or sedative procedures). We should focus in a different manner for each scenario.

(II) Define specific populations. We must define exactly which high-risk patients are potential candidates and would benefit more from awake procedures. We must also define baseline features (BMI, gender, preoperative FEV1 and DLCO, cTNM, etc.) for attempting anatomical resections such as lobectomies or segmentectomies in lowrisk ideal patients in order to avoid unnecessary risks.

(III) Objectives pursued. This is the cornerstone of research in nonintubated thoracic surgery: defining what we aim for and how we will assess it. For high-risk patients not considered for conventional anesthesia, we should define the target outcomes in terms of feasibility and complications. But for anatomical resections in low-risk ideal patients, we should analyze biological markers of stress and immune disbalance, in order to assess if nonintubated surgery is less disruptive for body homeostasis (serum cortisol, lymphocyte subpopulations, NK cells, etc.), and also variables regarding the quality of the oncological surgery (lymph node number and stations, resection margins). We should also analyze intra and postoperative complications, especially focused on safety levels of hypercapnia and which baseline features of the patients may predict the appearance of severe hypercapnia. Finally, we must compare postoperative parameters such as resumption of oral intake, chest tube duration or hospital stay in the same context of enhanced recovery programs both for study and control groups.

(IV) Investigate within multicenter groups. Nonintubated surgery remains a challenge for most thoracic surgeons and anesthesiologists, so special efforts should be made to create multidisciplinary (thoracic surgeons and anesthesiologists) and multicenter interest groups in order to collect significant case-load under homogeneous conditions. Without this critical analysis, maybe nonintubated segmentectomies and other anatomical resections will be limited to sporadic reports from very few specialized centers.

Efforts should be made in order to clarify the concepts and the targeted population, homogenize the anesthetic methods, design randomized trials assessing the variables where the expected benefits can be measured, and state 
conclusion according to these outcomes.

\section{Acknowledgments}

To our Thoracic Surgery residents, who enthusiastically believe in what we do and how we do it. There is no progress without passion, without mistakes, without selfcriticism. We must encourage them to keep enough humbleness to push forward but never miss our final target: patient's health and wellness.

Funding: None.

\section{Footnote}

Provenance and Peer Review: This article was commissioned by the Guest Editor (Michel Gonzalez) for the series "VATS Segmentectomy" published in Fournal of Visualized Surgery. The article has undergone external peer review.

Reporting Checklist: The authors have completed the Narrative Review reporting checklist. Available at https:// jovs.amegroups.com/article/view/10.21037/jovs-21-33/rc

Peer Review File: Available at https://jovs.amegroups.com/ article/view/10.21037/jovs-21-33/prf

Conflicts of Interest: All authors have completed the ICMJE uniform disclosure form (available at https://jovs. amegroups.com/article/view/10.21037/jovs-21-33/coif). The series "VATS Segmentectomy" was commissioned by the editorial office without any funding or sponsorship. The authors have no other conflicts of interest to declare.

Ethical Statement: The authors are accountable for all aspects of the work in ensuring that questions related to the accuracy or integrity of any part of the work are appropriately investigated and resolved.

Open Access Statement: This is an Open Access article distributed in accordance with the Creative Commons Attribution-NonCommercial-NoDerivs 4.0 International License (CC BY-NC-ND 4.0), which permits the noncommercial replication and distribution of the article with the strict proviso that no changes or edits are made and the original work is properly cited (including links to both the formal publication through the relevant DOI and the license). See: https://creativecommons.org/licenses/by-nc-nd/4.0/.

\section{References}

1. Ginsberg RJ, Rubinstein LV. Randomized trial of lobectomy versus limited resection for T1 N0 non-small cell lung cancer. Lung Cancer Study Group. Ann Thorac Surg 1995;60:615-22; discussion 622-3.

2. Field JK, deKoning H, Oudkerk M, et al. Implementation of lung cancer screening in Europe: challenges and potential solutions: summary of a multidisciplinary roundtable discussion. ESMO Open 2019;4:e000577.

3. Sands J, Tammemägi MC, Couraud S, et al. Lung Screening Benefits and Challenges: A Review of The Data and Outline for Implementation. J Thorac Oncol 2021;16:37-53.

4. de Koning HJ, van der Aalst CM, de Jong PA, et al. Reduced Lung-Cancer Mortality with Volume CT Screening in a Randomized Trial. N Engl J Med 2020;382:503-13.

5. Martin-Ucar AE, Delgado Roel M. Indication for VATS sublobar resections in early lung cancer. J Thorac Dis 2013;5 Suppl 3:S194-9.

6. Bao F, Ye P, Yang Y, et al. Segmentectomy or lobectomy for early stage lung cancer: a meta-analysis. Eur J Cardiothorac Surg 2014;46:1-7.

7. Liu Y, Huang C, Liu H, et al. Sublobectomy versus lobectomy for stage IA (T1a) non-small-cell lung cancer: a meta-analysis study. World J Surg Oncol 2014;12:138.

8. Dziedzic R, Zurek W, Marjanski T, et al. Stage I nonsmall-cell lung cancer: long-term results of lobectomy versus sublobar resection from the Polish National Lung Cancer Registry. Eur J Cardiothorac Surg 2017;52:363-9.

9. Ettinger DS, Wood DE, Aisner DL, et al. Non-Small Cell Lung - NCCN Clinical Practice Guidelines in Oncology, v6. NCCN Guidel. 2020.

10. Hung MH, Hsu HH, Chen KC, et al. Nonintubated thoracoscopic anatomical segmentectomy for lung tumors. Ann Thorac Surg 2013;96:1209-15.

11. Liu HY, Hsu HH, Tsai TM, et al. Nonintubated Versus Intubated Uniportal Thoracoscopic Segmentectomy for Lung Tumors. Ann Thorac Surg 2021;111:1182-9.

12. Hung MH, Cheng YJ, Hsu HH, et al. Nonintubated uniportal thoracoscopic segmentectomy for lung cancer. J Thorac Cardiovasc Surg 2014;148:e234-5.

13. Mineo TC, Tacconi F. Nonintubated thoracic surgery: a lead role or just a walk on part? Chin J Cancer Res 2014;26:507-10. 
14. Gálvez C, Bolufer S, Gálvez E, et al. Anatomic Segmentectomy in Nonintubated Video-Assisted Thoracoscopic Surgery. Thorac Surg Clin 2020;30:61-72.

15. Xue W, Duan G, Zhang X, et al. Comparison of nonintubated and intubated video-assisted thoracoscopic surgeries of major pulmonary resections for lung cancer-a meta-analysis. World J Surg Oncol 2021;19:87.

16. Liu HY, Chiang XH, Hung MH, et al. Nonintubated uniportal thoracoscopic segmentectomy for lung cancer. J Formos Med Assoc 2020;119:1396-404.

17. Chen KC, Cheng YJ, Hung MH, et al. Nonintubated thoracoscopic lung resection: a 3-year experience with 285 cases in a single institution. J Thorac Dis 2012;4:347-51.

18. Galvez C, Navarro-Martinez J, Bolufer S, et al. Nonintubated uniportal left-lower lobe upper segmentectomy (S6). J Vis Surg 2017;3:48.

19. Guo Z, Shao W, Yin W, et al. Analysis of feasibility and safety of complete video-assisted thoracoscopic resection of anatomic pulmonary segments under non-intubated anesthesia. J Thorac Dis 2014;6:37-44.

20. Wang ML, Galvez C, Chen JS, et al. Non-intubated single-incision video-assisted thoracic surgery: a two-center cohort of 188 patients. J Thorac Dis 2017;9:2587-98.

21. Guo Z, Yin W, Pan H, et al. Video-assisted thoracoscopic surgery segmentectomy by non-intubated or intubated anesthesia: a comparative analysis of short-term outcome. J Thorac Dis 2016;8:359-68.

22. Liu J, Cui F, Pompeo E, et al. The impact of nonintubated versus intubated anaesthesia on early outcomes of video-assisted thoracoscopic anatomical resection in non-small-cell lung cancer: a propensity score matching analysis. Eur J Cardiothorac Surg 2016;50:920-5.

23. Salati M, Brunelli A, Decaluwe H, et al. Report from the European Society of Thoracic Surgeons Database 2017: patterns of care and perioperative outcomes of surgery for malignant lung neoplasm. Eur J Cardiothorac Surg 2017;52:1041-8.

24. Vladimir C, Zdenek K, Lukas F, et al. Clarification of

doi: $10.21037 /$ jovs-21-33

Cite this article as: Galvez C, Bolufer S, Córcoles JM, Lirio F, Sesma J, Obeso A. Nonintubated anatomical segmentectomy: does the benefit overcome the risks?-a narrative review. J Vis Surg 2022;8:38. the resection line non-intubated segmentectomy using indocyanine green. Ann Transl Med 2019;7:38.

25. Wang ML, Hung MH, Hsu HH, et al. Non-intubated thoracoscopic surgery for lung cancer in patients with impaired pulmonary function. Ann Transl Med 2019;7:40.

26. Edwards JG, Chansky K, Van Schil P, et al. The IASLC Lung Cancer Staging Project: Analysis of Resection Margin Status and Proposals for Residual Tumor Descriptors for Non-Small Cell Lung Cancer. J Thorac Oncol 2020;15:344-59.

27. Postmus PE, Kerr KM, Oudkerk M, et al. Early and locally advanced non-small-cell lung cancer (NSCLC): ESMO Clinical Practice Guidelines for diagnosis, treatment and follow-up. Ann Oncol 2017;28:iv1-iv21.

28. Pompeo E, Rogliani P, Tacconi F, et al. Randomized comparison of awake nonresectional versus nonawake resectional lung volume reduction surgery. J Thorac Cardiovasc Surg 2012;143:47-54, 54.e1.

29. Pompeo E, Mineo TC. Awake operative videothoracoscopic pulmonary resections. Thorac Surg Clin 2008;18:311-20.

30. Guarracino F, Gemignani R, Pratesi G, et al. Awake palliative thoracic surgery in a high-risk patient: one-lung, non-invasive ventilation combined with epidural blockade. Anaesthesia 2008;63:761-3.

31. Rocco G, Romano V, Accardo R, et al. Awake singleaccess (uniportal) video-assisted thoracoscopic surgery for peripheral pulmonary nodules in a complete ambulatory setting. Ann Thorac Surg 2010;89:1625-7.

32. Pompeo E, Mineo TC. Awake pulmonary metastasectomy. J Thorac Cardiovasc Surg 2007;133:960-6.

33. Liu S, Mao Y, Qiu P, et al. Airway Rupture Caused by Double-Lumen Tubes: A Review of 187 Cases. Anesth Analg 2020;131:1485-90.

34. Tacconi F, Vanni G, Pompeo E. Systemic host response in awake thoracic surgery. Available online: https://www. eurekaselect.com/51031/chapter/systemic-host-responsein-awake-thoracic-surgery 


\section{Supplementary}

Table S1 Main outcomes of awake thoracic surgery from comparative studies

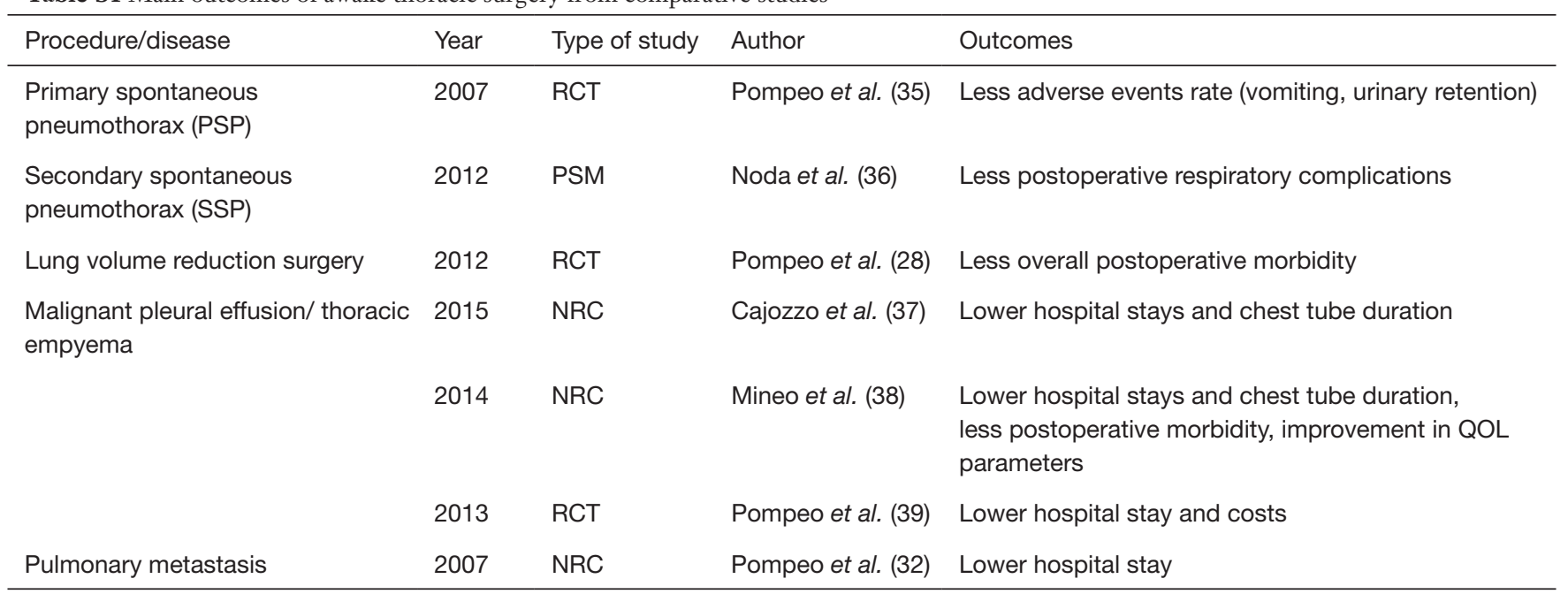

PSM, propensity score matching; RCT, randomized clinical trial; NRC, non-randomized comparative; QOL, quality of life.

\section{References}

35. Pompeo E, Tacconi F, Mineo D, et al. The role of awake video-assisted thoracoscopic surgery in spontaneous pneumothorax. J Thorac Cardiovasc Surg 2007;133:786-90.

36. Noda M, Okada Y, Maeda S, et al. Is there a benefit of awake thoracoscopic surgery in patients with secondary spontaneous pneumothorax? J Thorac Cardiovasc Surg 2012;143:613-6.

37. Cajozzo M, Lo Iacono G, Raffaele F, et al. Thoracoscopy in pleural effusion--two techniques: awake single-access video-assisted thoracic surgery versus 2-ports video-assisted thoracic surgery under general anesthesia. Future Oncol 2015;11:39-41.

38. Mineo TC, Sellitri F, Tacconi F, et al. Quality of life and outcomes after nonintubated versus intubated video-thoracoscopic pleurodesis for malignant pleural effusion: comparison by a case-matched study. J Palliat Med 2014;17:761-8.

39. Pompeo E, Dauri M; Awake Thoracic Surgery Research Group. Is there any benefit in using awake anesthesia with thoracic epidural in thoracoscopic talc pleurodesis? J Thorac Cardiovasc Surg 2013;146:495-7.e1. 